Check for updates

Cite this: Phys. Chem. Chem. Phys., 2017, 19, 28963

Received 24th August 2017 Accepted 6th October 2017

DOI: $10.1039 / c 7 c p 05793 h$

rsc.li/pccp

\section{The interaction of halogen atoms and molecules with borophene $\dagger$}

\author{
Jamoliddin Khanifaev, ${ }^{a}$ Rengin Peköz, ${ }^{\mathrm{b}}$ Mine Konuk $^{\mathrm{a}}$ and Engin Durgun (D) *a
}

\begin{abstract}
The realization of buckled monolayer sheets of boron (i.e., borophene) and its other polymorphs has attracted significant interest in the field of two-dimensional systems. Motivated by borophene's tendency to donate electrons, we analyzed the interaction of single halogen atoms $(\mathrm{F}, \mathrm{Cl}, \mathrm{Br}, \mathrm{I})$ with borophene. The possible adsorption sites are tested and the top of the boron atom is found as the ground state configuration. The nature of bonding and strong chemical interaction is revealed by using projected density of states and charge difference analysis. The migration of single halogen atoms on the surface of borophene is analyzed and high diffusion barriers that decrease with atomic size are obtained. The metallicity of borophene is preserved upon adsorption but anisotropy in electrical conductivity is altered. The variation of adsorption and formation energy, interatomic distance, charge transfer, diffusion barriers, and bonding character with the type of halogen atom are explored and trends are revealed. Lastly, the adsorption of halogen molecules $\left(\mathrm{F}_{2}, \mathrm{Cl}_{2}, \mathrm{Br}_{2}, \mathrm{I}_{2}\right)$, including the possibility of dissociation, is studied. The obtained results are not only substantial for fundamental understanding of halogenated derivatives of borophene, but also are useful for near future technological applications.
\end{abstract}

\section{Introduction}

Following the synthesis of graphene, ${ }^{1}$ an immense amount of research has been conducted in the field of two dimensional (2D) materials. Since then various elemental 2D materials including silicene, ${ }^{2}$ germanene ${ }^{3}$ and stanene ${ }^{4}$ in group IV, and phosphorene, ${ }^{5}$ antimonene, ${ }^{6}$ and bismuthene ${ }^{7}$ in group $\mathrm{V}$ have been realized experimentally and also investigated in detail by advanced computational methods. The synthesis of borophene, ${ }^{8,9}$ a monoatomic lattice of boron atoms, has extended this family to group III elements.

Boron is a light element which is located at the boundary between metals and nonmetals in the periodic table and has a rich chemistry. Due to its electron shell configuration and flexibility to adopt $\mathrm{sp}^{2}$ hybridization, it can form complex bonds ranging from two-center-two electron to seven-centertwo electron bonds. ${ }^{10}$ This results in a variety of boron allotropes, which can be found in all-dimensions with diverse chemical and physical properties. ${ }^{11-15}$ In addition to various low-dimensional

\footnotetext{
${ }^{a}$ UNAM - National Nanotechnology Research Center and Institute of Materials Science and Nanotechnology, Bilkent University, 06800 Ankara, Turkey. E-mail: durgun@unam.bilkent.edu.tr

${ }^{b}$ Department of Electrical and Electronics Engineering, Attlim University, 06836 Ankara, Turkey

$\dagger$ Electronic supplementary information (ESI) available: Orbital decomposed projected density of states (PDOS) of all halogen atoms and first, second, and third nearest B atoms; the final configurations of adsorption of halogen molecules on borophene. See DOI: 10.1039/c7cp05793h
}

allotropes, very recently Mannix et $a{ }^{8}{ }^{8}$ have grown the first atomically thin boron sheet on an $\mathrm{Ag}(111)$ substrate under ultrahigh-vacuum conditions. It has a buckled structure with triangular arrangement belonging to the Pmmn space group. Following this work, two planar structures of borophene, namely $\beta_{12}$ and $\chi_{3}$ phases $^{9}$ were also synthesized. Both $\beta_{12}$ and $\chi_{3}$ sheets contain a periodic arrangement of holes which act as scavengers of electrons occupying antibonding bands, thus hexagonal holes improve the stability of borophene and contribute to the planarity of the structure. ${ }^{16}$ In addition to these, various stable borophene polymorphs with different hole patterns and densities have also been theoretically predicted ${ }^{12,17}$ which may be realized in the near future.

Even though all known bulk boron allotropes are semiconductors at ambient conditions, buckled borophene is metallic with highly anisotropic electronic properties. ${ }^{8}$ It also possesses mechanical anisotropy where the in-plane Young's modulus is equal to $170 \mathrm{GPa}$ nm along the corrugated (zigzag) direction, and $398 \mathrm{GPa} \mathrm{nm}$ along the uncorrugated (armchair) direction, which is even higher than that obtained for graphene (340 GPa nm). ${ }^{18}$ The structure has negative Poisson's ratio due to the out-of-plane buckling $^{8}$ and also significant negative thermal expansion coefficients are reported for both directions. ${ }^{19}$ In a theoretical study, large optical anisotropy with high optical transparency and electrical conductivity is reported. ${ }^{20}$ Furthermore, a systematic investigation of thermal properties indicates that the thermal conductivity of borophene is also anisotropic and low due to the strong phonon-phonon scattering. The calculated values are 
$\sim 20 \times 10^{-9} \mathrm{~W} \mathrm{~K}^{-1}$ and $\sim 40 \times 10^{-9} \mathrm{~W} \mathrm{~K}^{-1}$, along the zigzag and armchair directions, respectively. ${ }^{19}$ Moreover, experimentally realized borophene sheets are predicted to display intrinsic superconducting behavior at low temperatures ${ }^{21,22}$ which can be enhanced by the tensile strain and hole doping. ${ }^{23}$

These novel properties make borophene an ideal candidate for various applications. ${ }^{9} \mathrm{Ab}$ initio studies predicted that doped borophene can be used as a catalyst for hydrogen and oxygen evolution reactions, ${ }^{24}$ is a promising anode material for lithium-ion batteries, ${ }^{25,26}$ and is a potential hydrogen storage medium. ${ }^{27}$ Boronbased microelectronic device applications is an another interesting field, however, the implementation of borophene requires further investigation. This can be possible by functionalization of such systems in order to attain desired properties. An extensive amount of experimental and theoretical work dedicated to the doping of $2 \mathrm{D}$ materials has proved that doping is an effective and upcoming technique for tuning the existing properties. ${ }^{28-31}$

In this respect, halogen atoms and molecules are an interesting class of adsorbates due to their high electronegativity. They can substantially modify the structural and electronic features of $2 \mathrm{D}$ materials upon adsorption or decoration, and they are widely studied both experimentally and theoretically. For instance, fully and partially fluorinated graphene has already been synthesized ${ }^{32}$ and explored in detail. ${ }^{33,34}$ It was shown that fluorine coverage results in band gap opening which can be engineered with the degree of fluorination. ${ }^{35}$ Moreover, partially chlorinated ${ }^{36}$ and brominated $^{37}$ graphene derivatives have been reported and the interaction of halogen atoms with graphene has been studied by first-principles methods. ${ }^{33,38-40}$ Not only graphene but also chlorinated silicene ${ }^{41}$ and fluorinated boron nitride sheets ${ }^{42}$ have also been realized. In addition to experimental efforts, various theoretical studies analyzed the interaction of halogen atoms with $2 \mathrm{D}$ systems. $^{43-45}$ It is reported that halogenated 2D materials exhibit remarkable electronic, optical, thermal, mechanical, and chemical properties in comparison with bare counterparts.

Motivated by these studies, we investigate the interaction of single halogen atoms $(\mathrm{X}=\mathrm{F}, \mathrm{Cl}, \mathrm{Br}, \mathrm{I})$ with a buckled monolayer sheet of boron (which will be referred simply as borophene) by using $a b$ initio methods. Firstly, we test all the possible adsorption sites and determine the ground state configurations. The nature of bonding is revealed by projected density of states (PDOS) and charge transfer analyses. The migration of single halogen atoms on the surface of borophene is studied and diffusion barriers are calculated. The change in the electronic structure is also examined and compared with pristine borophene. The variation of adsorption energy, interatomic distance, charge transfer, diffusion barriers, and bonding character with the type of halogen (i.e. up/down in group VII) is explored and trends are revealed. Finally, the adsorption of halogen molecules $\left(\mathrm{X}_{2}=\mathrm{F}_{2}, \mathrm{Cl}_{2}, \mathrm{Br}_{2}, \mathrm{I}_{2}\right)$ and the case of dissociation are studied.

\section{Computational methodology}

We perform ground-state total energy and electronic structure calculations by first-principles methods based on density functional theory (DFT). ${ }^{46,47}$ The projector augmented-wave potential (PAW) ${ }^{48,49}$ with an energy cutoff at $520 \mathrm{eV}$ is used as implemented in the Vienna ab initio simulation package (VASP). ${ }^{50,51}$ The electron exchange and correlation potential is described by the generalized gradient approximation (GGA) in Perdew-Burke-Ernzerhof (PBE) form. ${ }^{52}$ All atomic positions are optimized by minimizing the total energy and atomic forces using the conjugate gradient method by setting energy and force convergence to $10^{-5} \mathrm{eV}$ and $10^{-2} \mathrm{eV} \AA^{-1}$, respectively. The van der Waals (vdW) interactions are taken into account by using the DFT-D2 method for the case of molecular adsorption. ${ }^{53}$ Bader analysis is implemented ${ }^{54}$ to quantify the charge exchange between the $\mathrm{X}$ atoms and the borophene. To avoid interactions between dopants, a $7 \times 4$ supercell is used with lattice constants, $11.45 \AA$ and $11.30 \AA$ in the $x$ and $y$ directions, respectively, and $15 \AA$ vacuum space is adopted in the non-periodic $z$-direction. The Brillouin zone (BZ) is sampled by a $\Gamma$-centered $6 \times 6 k$-point mesh. ${ }^{55}$

The adsorption (or binding) and formation energies of halogen atoms are obtained by using the formulas:

$$
E_{\mathrm{b}}=E_{\mathrm{T}}(\text { borophene })+E_{\mathrm{T}}(\mathrm{X})-E_{\mathrm{T}}(\text { borophene }+\mathrm{X})
$$

and

$$
E_{\mathrm{f}}=E_{\mathrm{T}}(\text { borophene })+E_{\mathrm{T}}\left(\mathrm{X}_{2}\right) / 2-E_{\mathrm{T}}(\text { borophene }+\mathrm{X})
$$

where $E_{\mathrm{T}}$ (borophene), $E_{\mathrm{T}}(\mathrm{X}), E_{\mathrm{T}}\left(\mathrm{X}_{2}\right)$, and $E_{T}$ (borophene $\left.+\mathrm{X}\right)$ are the total energies of pristine borophene, free $\mathrm{X}$ atoms, $\mathrm{X}_{2}$ molecules and the doped system, respectively. ${ }^{56,57}$ All energies are calculated by using the same supercell size and identical parameters. A positive value of $E_{\mathrm{b}}$ implies that adsorption is energetically favorable. The diffusion path calculations are performed using the nudged-elastic band (NEB) approach where intermediate images along the reaction path are optimized while equal spacing to neighboring images is maintained. ${ }^{58,59}$

\section{Results and discussion}

The adsorption of halogen atoms can substantially modify the physical and chemical properties of borophene and enhance the stability of the system by accepting the electrons from antibonding states. ${ }^{8}$ Revealing the process of doping is also essential for plausible halogenated derivatives of borophene. To study the single halogen atom (X: F, Cl, Br, I) adsoption, we start with structural optimization of monolayer borophene. The optimized lattice constants of pristine borophene are calculated as $a=1.62 \AA$ and $b=2.86 \AA$ with a buckling of $0.90 \AA$ which are in agreement with previous studies. ${ }^{8,60}$ The coupling between $\mathrm{X}$ atoms is minimized by using a $7 \times 4$ borophene supercell (with lattice parameters of $11.30 \AA$ and $11.45 \AA$ ) which is tested to be sufficiently long enough in both directions. This system, as shown in Fig. 1, contains $56 \mathrm{~B}$ and $1 \mathrm{X}$ atoms which is equivalent to the halogen concentration of $1.75 \%$.

Four possible sites are considered for adsorption as shown in Fig. 1: top of the $B$ atom on the upper $\left(T_{u}\right)$ or lower layer $\left(T_{d}\right)$, and above the bridge of successive $\mathrm{B}$ atoms on the upper $\left(\mathrm{B}_{\mathrm{u}}\right)$ or 
(a)

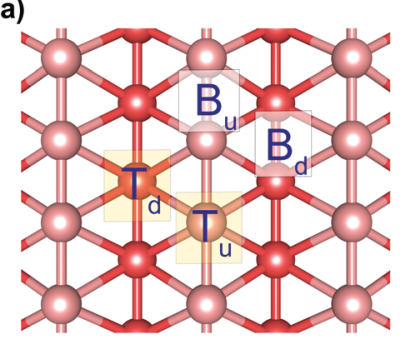

(c)

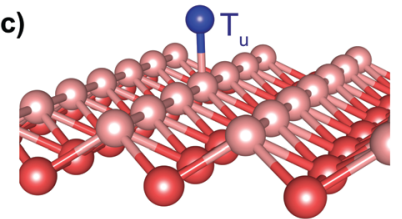

(b)

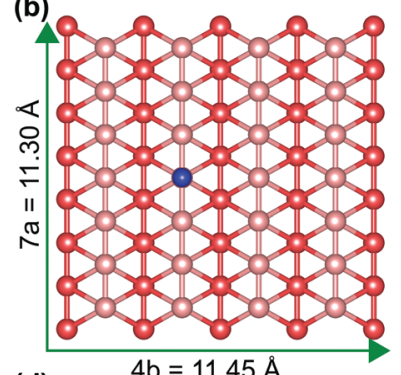

(d)

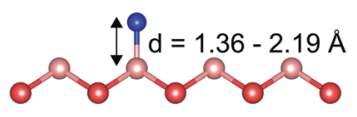

Fig. 1 (a) The possible adsorption sites on borophene sheets: top of the $B$ atom on the upper $\left(T_{u}\right)$ or lower layer $\left(T_{d}\right)$, and above the middle of the successive $B$ atoms on the upper $\left(B_{u}\right)$ or lower layer $\left(B_{d}\right)$ (b). (c) Top perspective and (d) side view of the $7 \times 4$ supercell with a single adatom ( $\mathrm{X}: \mathrm{F}, \mathrm{Cl}, \mathrm{Br}, \mathrm{I}$ ) on the $\mathrm{T}_{\mathrm{u}}$ site. The boron atom on the upper (lower) layer and halogen atoms are shown by light (dark) red and blue spheres, respectively.

lower layer $\left(B_{d}\right)$. Our results indicate that only $T_{u}$ and $B_{d}$ are stable adsorption sites and adatoms on $T_{d}$ and $B_{u}$ slide to $T_{u}$ which is the ground state configuration for all $\mathrm{X}$ atoms. The top site is also favored for halogen atoms adsorbed on monolayers of group IV elements. ${ }^{34,38,43-45}$ Adsorption energy $\left(E_{\mathrm{b}}\right)$, formation energy $\left(E_{\mathrm{f}}\right)$, dissociation energy of a halogen molecule $\left(E_{\mathrm{dis}}\right)$, bonding distance between $\mathrm{B}$ and $\mathrm{X}$ atoms $\left(d_{\mathrm{B}-\mathrm{X}}\right)$, and the amount of charge transferred to $\mathrm{X}$ atoms $\left(\rho_{\mathrm{X}}\right)$ for the ground state configuration $\left(\mathrm{T}_{\mathrm{u}}\right.$ site) are summarized in Table $1 . E_{\mathrm{b}}$ for the other stable site, $\mathrm{B}_{\mathrm{d}}$ is also given in parentheses for comparison.

Upon adsorption, all $\mathrm{X}$ atoms slightly distort the pristine borophene by attracting the underlying $\mathrm{B}$ atom at $\mathrm{T}_{\mathrm{u}}$. The amount of distortion decreases from $\mathrm{F}$ to I atoms. The calculated $E_{\mathrm{b}}$ is high for all $\mathrm{X}$ atoms indicating a strong chemical binding. At $B_{d}$, each $X$ atom attracts two $B$ atoms at $T_{u}$ and makes bonds with both of them. As will be discussed later in this section, this configuration is fragile and can only be stable at low temperatures. The highest $E_{\mathrm{b}}$ is obtained for $\mathrm{F}$ which is $5.03 \mathrm{eV}$ and decreases gradually to $2.09 \mathrm{eV}$ down the group. In addition, all calculated formation energies are positive indicating that the adsorption of halogen atoms on borophene is an exothermic process. The calculated $d_{\mathrm{B}-\mathrm{X}}$ is only slightly larger than those reported for boron trihalides, thus the bonding characters are expected to

Table 1 Adsorption energy $\left(E_{b}\right)$, formation energy $\left(E_{f}\right)$, dissociation energy of a halogen molecule $\left(E_{\text {dis }}\right)$, bonding distance between $B$ and $X$ atoms $\left(d_{\mathrm{B}-\mathrm{X}}\right)$, electronegativity difference between $\mathrm{B}$ and $\mathrm{X}$ atoms $(\Delta \chi)$ and amount of charge transferred to $X$ atoms $\left(\rho_{X}\right)$ are given for the ground state configuration ( $T_{\mathrm{u}}$ site). $E_{\mathrm{b}}$ for the other stable site, $B_{\mathrm{d}}$, is given in parentheses. All energies are given per atom

\begin{tabular}{lllllll}
\hline Atom & $E_{\mathrm{b}}[\mathrm{eV}]$ & $E_{\mathrm{f}}[\mathrm{eV}]$ & $E_{\mathrm{dis}}[\mathrm{eV}]$ & $d_{\mathrm{B}-\mathrm{X}}[\AA]$ & $\Delta \chi$ & $\rho_{\mathrm{X}}[e]$ \\
\hline $\mathrm{F}$ & $5.03(4.08)$ & 3.87 & -1.15 & 1.36 & 1.94 & -0.79 \\
$\mathrm{Cl}$ & $3.23(2.67)$ & 1.81 & -1.42 & 1.79 & 1.12 & -0.63 \\
$\mathrm{Br}$ & $2.64(2.17)$ & 1.41 & -1.23 & 1.97 & 0.92 & -0.48 \\
$\mathrm{I}$ & $2.09(1.76)$ & 0.98 & -1.12 & 2.19 & 0.66 & -0.14
\end{tabular}

(a)

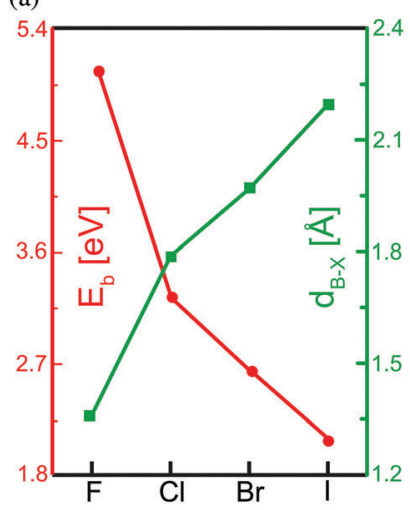

(b)

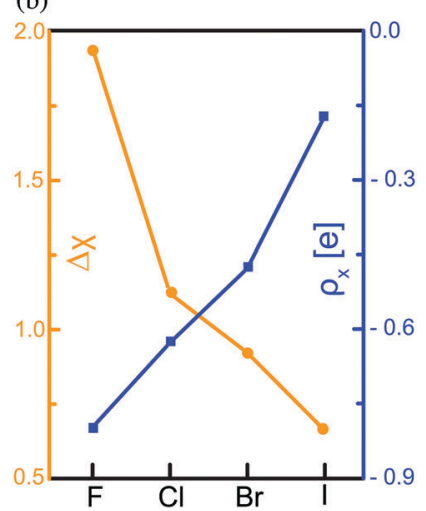

Fig. 2 The variation of (a) $E_{\mathrm{b}}$ (red line) and $d_{\mathrm{B}-\mathrm{x}}$ (green line), and (b) $\Delta \chi$ (orange line) and $\rho_{X}$ (blue line) with the type of $X$ atom.

be analogous. Not suprisingly, $d_{\mathrm{B}-\mathrm{x}}$ shortens with increasing $E_{\mathrm{b}}$ indicating a stronger bond. The variations of $E_{\mathrm{b}}$ and $d_{\mathrm{B}-\mathrm{X}}$ with the type of $\mathrm{X}$ atom are shown in Fig. 2. The strength of binding can also be linked to the amount of charge transfer from borophene to $\mathrm{X}$ atoms. The Bader analyses demonstrate that while $\mathrm{F}$ accepts $-0.79|e|$ (negative value indicates that charge is accepted by $\mathrm{X}$ atoms), it is only $-0.14|e|$ for I. $\rho_{\mathrm{X}}$ decreases down the group following the same trend of $\Delta \chi$ as illustrated in Fig. 2.

The calculated $E_{\mathrm{b}}$ values are significantly higher than those obtained for interaction of $\mathrm{X}$ atoms with group IV sheets. For instance, $E_{\mathrm{b}}$ is reported as $2.71 \mathrm{eV},{ }^{34} 1.13 \mathrm{eV},{ }^{38}$ and $0.86^{40} \mathrm{eV}$ for $\mathrm{F}, \mathrm{Cl}$, and I adsorption on graphene, respectively. Interestingly, other widely used adatoms such as $\mathrm{H}, \mathrm{Li}$ and $\mathrm{O}$ are also strongly bound to borophene with $E_{\mathrm{b}}$ of $2.99 \mathrm{eV},{ }^{61} 2.84 \mathrm{eV}^{61}$ and $4.55 \mathrm{eV},{ }^{60}$ respectively. These results also indicate that borophene has a very reactive surface.

The lateral diffusion of $\mathrm{X}$ atoms on the borophene surface from $T_{u}$ to the first nearest $T_{u}\left(T_{u} \rightarrow T_{u}\right)$ and then to the next nearest $B_{d}\left(T_{u} \rightarrow B_{d}\right)$ is calculated by using the NEB method. ${ }^{58,59}$ The variation of total energy along this path together with relevant energy barriers for $\mathrm{Cl}$ atom is shown in Fig. 3(a) as an example. Two significant barriers are noticed: along $T_{u} \rightarrow T_{u}$ $\left(\mathrm{Q}_{1}\right)$ and along $\mathrm{T}_{\mathrm{u}} \rightarrow \mathrm{B}_{\mathrm{d}}\left(\mathrm{Q}_{2}\right)$. Both $\mathrm{Q}_{1}$ and $\mathrm{Q}_{2}$ are high and this lowers the possibility of migration of $\mathrm{X}$ atoms on the surface. The calculated diffusion barriers are substantially higher than those obtained for graphene where migration occurs with almost no barrier. ${ }^{38}$ On the other hand, the barrier is very low along $B_{d} \rightarrow T_{u}$ for all cases $\left(Q_{3}<60 \mathrm{meV}\right)$ indicating that the $B_{d}$ site is stable only at low temperatures, and $\mathrm{X}$ atoms remain bound only to $\mathrm{T}_{\mathrm{u}}$ at ambient conditions. $\mathrm{Q}_{1}$ and $\mathrm{Q}_{2}$ decrease down in the group as illustrated in Fig. 3(b) and the highest energy barrier is obtained for $\mathrm{F}$.

Next, we analyze the nature of bonding between $\mathrm{X}$ atoms and borophene. While B atoms have three valence electrons, each of them has six nearest neighbors in buckled triangular borophene. Accordingly, unlike 2D group IV systems, some of the in-plane $\mathrm{sp}^{2}$ antibonding states are occupied ${ }^{62}$ and borophene has a tendency to donate electrons. Taking the high $\chi$ of halogens into account, 


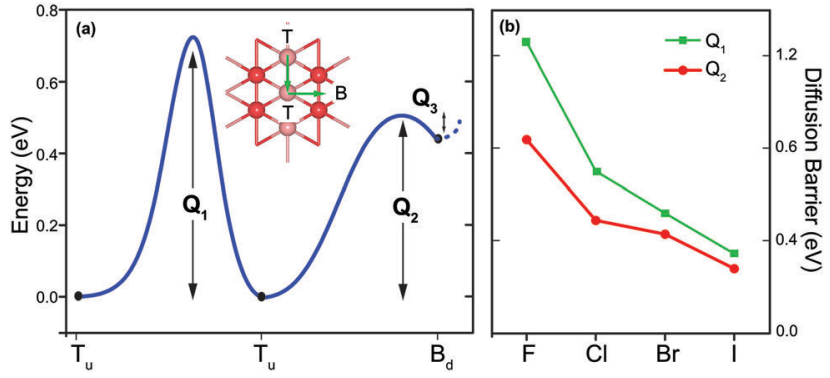

Fig. 3 (a) The variation of the total energy along the diffusion path together with relevant energy barriers for $\mathrm{Cl}$ atoms. Zero of energy is set to the ground state energy at $T_{u}$. The diffusion path is shown as an inset (b) The variation of diffusion barriers, $Q_{1}$ (from $T_{u}$ to $T_{u}$ ) and $Q_{2}$ (from $T_{u}$ to $B_{d}$ ) with the type of halogen atoms.

charge transfer from borophene to $\mathrm{X}$ atoms upon adsorption as given in Table 1 is expected. $\rho_{\mathrm{X}}$ is the highest for the case of $\mathrm{F}$ atom. The charge difference analyses indicate that the transferred charge is not only accumulated on the $\mathrm{F}$ atom but also between the $\mathrm{F}-\mathrm{B}$ bond. The charge is depleted from the nearby $\mathrm{B}$ atoms as shown in Fig. $4(\mathrm{a})$. As $\Delta \chi$ between $\mathrm{F}$ and B is high $(\Delta \chi \sim 2)$, the bonding has mainly an ionic character. Interestingly, a recent study reported that an adsorbed $\mathrm{H}$ atom also extracts charge from borophene and the amount is calculated as $-0.72|e|$, which is comparable to the case of $\mathrm{F}$ atoms. ${ }^{61,63}$ The charge profile is similar for other halogen atoms as well. As discussed above, $\rho_{\mathrm{X}}$ is correlated with $\Delta \chi$ (Fig. 2(b)) and thus decreases down the group which is also noticeable from the charge difference analysis shown in Fig. 4(b-d). Different from the F case, charge is
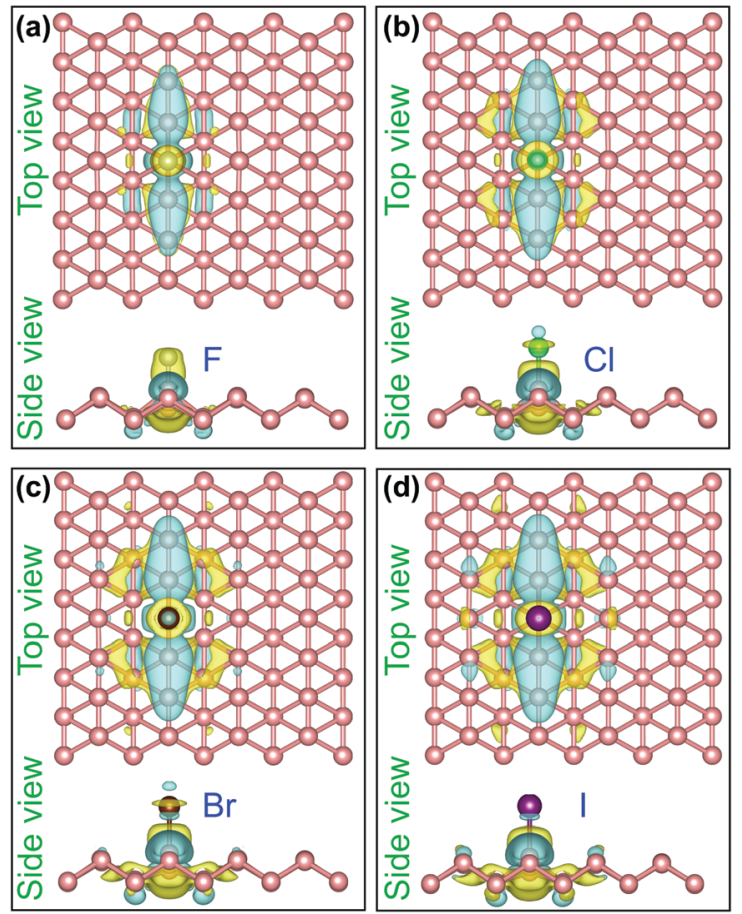

Fig. 4 The charge density difference profiles of (a) $\mathrm{F}$, (b) $\mathrm{Cl}$, (c) Br, and (d) I doped borophene for a $7 \times 4$ supercell. Yellow and blue colors represent charge accumulation and depletion, respectively. accumulated more on the $\mathrm{B}-\mathrm{X}$ bond for other halogen atoms and bonding has more covalent character.

In general, in-plane bonds resulting from $\mathrm{sp}^{2}$ hybridization are stronger than out-of-plane bonds. This makes buckled triangular borophene less stable than planar $\alpha$ - and $\beta$-borophene polymorphs where two- and three-center bondings are balanced. Therefore, halogen atoms which act as electron acceptors, can extract excess electrons occupying antibonding states which enhances the stability of buckled borophene.

Following this discussion, we also analyzed the projected density of states (PDOS) to reveal the orbital contributions to bonding. The PDOS of the in-plane and out-of-plane states for the $\mathrm{X}$ and $\mathrm{B}$ atom on $\mathrm{T}_{\mathrm{u}}\left(\mathrm{B}^{1}\right)$ are shown in Fig. 5 and the contributions from the second and the third nearest $\mathrm{B}$ atoms $\left(\mathrm{B}^{2}\right.$ and $\left.\mathrm{B}^{3}\right)$ are given in Fig. S1, ESI. $\dagger$ Our results indicate that
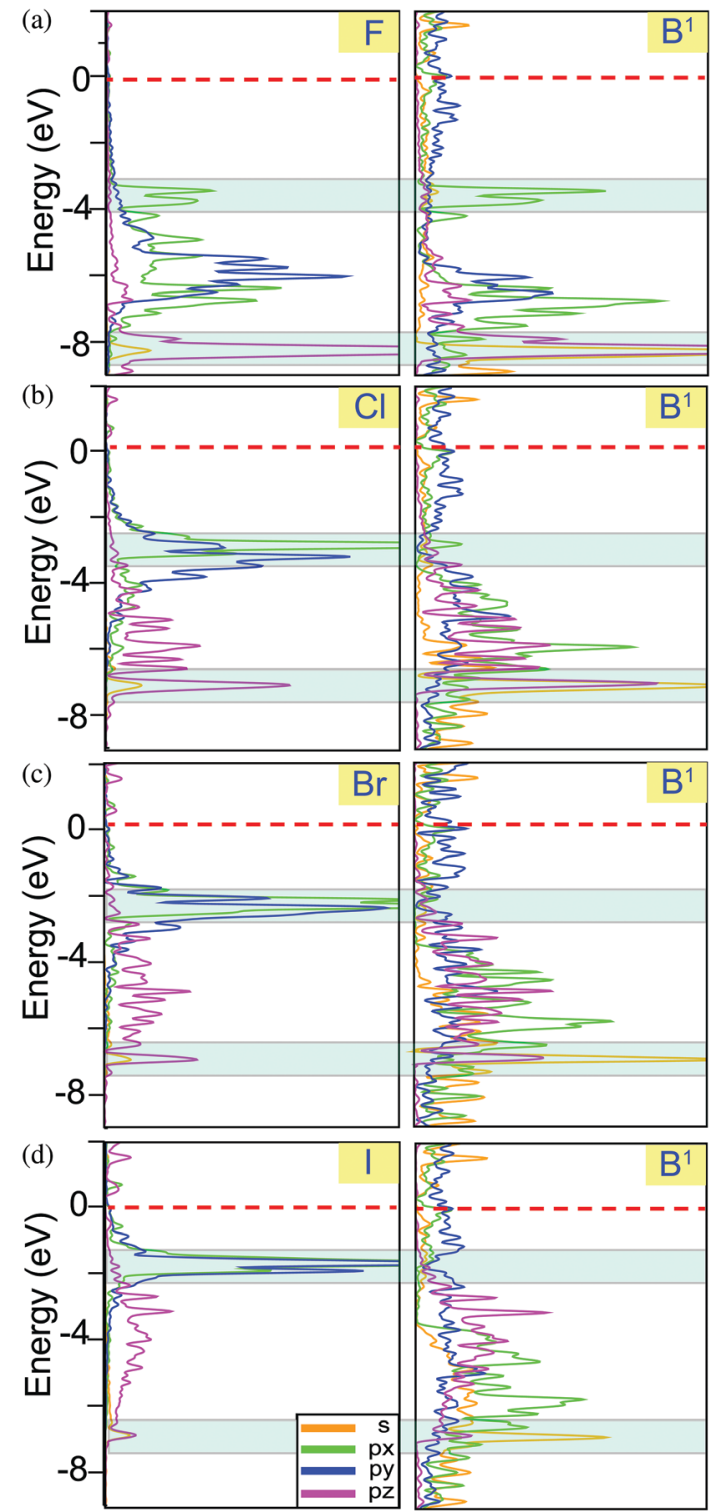

Fig. 5 Orbital decomposed projected density of states (PDOS) of (a) F, (b) $\mathrm{Cl}$, (c) $\mathrm{Br}$, and (d) I and the first nearest $\mathrm{B}$ atom at the $\mathrm{T}_{\mathrm{u}}$ site $\left(\mathrm{B}^{1}\right)$. Main peaks are highlighted with light green. 
while $\mathrm{Cl}, \mathrm{Br}$, and I have similar bonding character, $\mathrm{F}$ differs from them. Upon adsorption, the bonding states of $\mathrm{F}$ and $\mathrm{B}$ at $\mathrm{T}_{\mathrm{u}}$ are occupied and bonding is mainly formed from hybridization of the $\mathrm{p}_{z}$ orbital of $\mathrm{F}$, and $\mathrm{s}-\mathrm{p}_{z}$ orbitals of $\mathrm{B}^{1}$. This state is at $-8.2 \mathrm{eV}$ where Fermi level $\left(E_{\mathrm{F}}\right)$ is set at $0 \mathrm{eV}$. Another bonding state ( $\pi$ bonding) can be noticed at $-3.5 \mathrm{eV}$ upon hybridization of $p_{x}$ orbitals and not only $\mathrm{B}^{1}$ but $\mathrm{B}^{2}$ and $\mathrm{B}^{3}$ atoms contribute as well. For other $\mathrm{X}$ atoms $(\mathrm{Br}, \mathrm{Cl}$, and $\mathrm{I})$, the $\mathrm{s}-\mathrm{p}_{z}$ hybridized state is localized at $\sim-7 \mathrm{eV}$. While the contribution from the $\mathrm{p}_{z}$ orbital of $\mathrm{X}$ and $\mathrm{B}^{1}$ reduces down in the group, it increases for $\mathrm{B}^{2}$ and $\mathrm{B}^{3}$. Other bonding states due to hybridization of $\mathrm{p}_{x}$ orbitals weaken progressively with size and almost diminish for I. This level is $-1.9 \mathrm{eV}$ for I and deepens moving up to $\mathrm{F}$. The small atomic size and high $\chi$ of fluorine mainly account for the differences between $\mathrm{F}$ and other halogen atoms.

Lastly, we analyzed the electronic structure of X-doped systems by calculating the band structures. The pristine borophene is metallic with bands crossing $E_{\mathrm{F}}$ only along the directions parallel to the uncorrugated (armchair) direction. This results in an anisotropy and the electrical conductivity is confined only along this direction. The band structures of X-doped systems as shown in Fig. 6 indicate that $\mathrm{X}$ atoms not only generate deep localized states (Fig. 5), but modify the electronic structure. Upon adsorption, bands cross the $E_{\mathrm{F}}$ along all the directions and it makes the system more isotropic. Furthermore, an enhancement in electrical conductivity can be expected due to the increased number bands crossing the $E_{\mathrm{F}}$. This modification is related to structural distortion and change in the charge distribution (Fig. 4). Another interesting feature of the band profile is the band crossing at the $S$-symmetry point for the F-doped system. Similar crossing is also obtained for other $\mathrm{X}$ atoms but slightly below $E_{\mathrm{F}}$. These results indicate that the electronic properties of borophene can be modified upon passivation with halogen atoms, suggesting a new material similar to hydrogenated borophene ${ }^{63}$ or halogenated group IV systems. $^{38,39,64}$

Due to their high reactivity, halogens naturally occur in molecular form $\left(\mathrm{X}_{2}\right)$ with relatively low intermolecular bond energy when compared to other diatomic molecules (e.g. $\mathrm{H}_{2}$, $\mathrm{O}_{2}, \mathrm{~N}_{2}$ ) or their compounds with other atoms (e.g. $\mathrm{HX}, \mathrm{CX}_{4}$ ). Interaction of $\mathrm{X}_{2}$ molecules with group IV systems has been studied extensively ${ }^{40,65,66}$ and they are considered as molecular impurities. They generally interact weakly with 2D sheets and are physisorbed in molecular form. In a similar manner, together with single atom adsorption, we studied the interaction

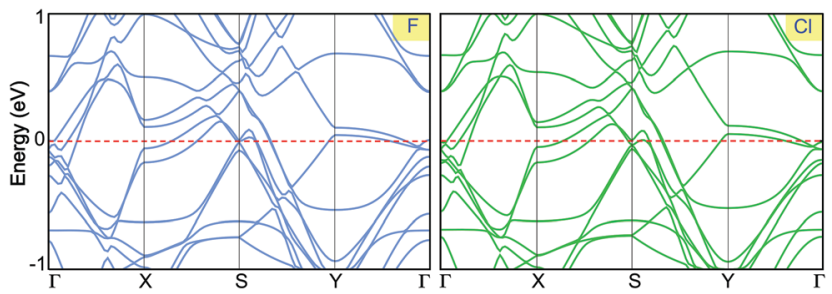

Fig. 6 The band structures of F- (left) and $\mathrm{Cl}$-doped (right) borophene along high-symmetry directions. The Fermi level is set to zero and is shown by a dashed red line.

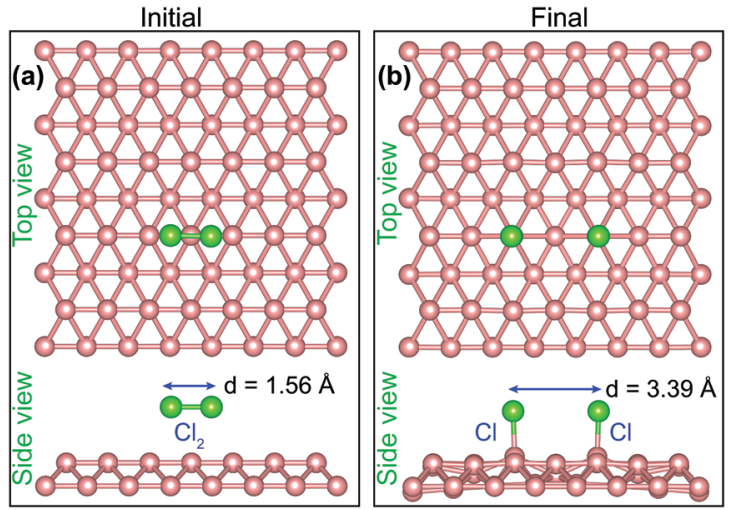

Fig. 7 (a) Initial and (b) final configurations of the top and side view of $\mathrm{Cl}_{2}$ adsorption on the borophene sheet. All halogen molecules dissociate into separate atoms as in the case of $\mathrm{Cl}_{2}$.

of $\mathrm{X}_{2}$ molecules with borophene. We tested various sites with different parallel and vertical orientations. Interestingly, for all cases $\mathrm{X}_{2}$ dissociates spontaneously without an activation barrier and is atomically adsorbed on the nearest $\mathrm{T}_{\mathrm{u}}$ sites. The dissociation energy $\left(E_{\mathrm{dis}}\right)$ for $\mathrm{X}_{2}$ molecules under vacuum is given in Table 1 . The parallel adsorption case for $\mathrm{Cl}_{2}$, which has the highest molecular bonding energy among halogens, is shown in Fig. 7 as a prototype. Other configurations are provided in Fig. S2, ESI. $\dagger \mathrm{X}_{2}$ also dissociates for perpendicular adsorption but this time while one of the $\mathrm{X}$ atoms binds to the $\mathrm{B}$ atom at the $\mathrm{T}_{\mathrm{u}}$ site, the other one ascends. Dissociation can be explained by the charge transfer from $\mathrm{B}$ to $\mathrm{X}$ atoms which weakens the $\mathrm{X}-\mathrm{X}$ interaction. Spontaneous dissociation of $\mathrm{X}_{2}$ molecules suggests fast adsorption rates ${ }^{67}$ and also indicates the high reactivity of the borophene sheet.

\section{Conclusions}

In conclusion, we systematically studied the interaction of halogen atoms with buckled borophene by means of first-principles methods. We tested all possible adsorption sites and determined that the ground state configuration is the top of the $\mathrm{B}$ atom in the upper layer in parallel with the results obtained for monolayers of group IV elements. Upon adsorption all halogen atoms extract a substantial amount of charge from the boron sheet. The amount of charge transfer decreases from $\mathrm{F}$ to I atoms, which is correlated with the change in the electronegativity that progressively decreases with size. The charge transfer also determines the strength of binding and halogen atoms are strongly bound to borophene. The obtained energy barriers along the diffusion paths are notably high indicating low possibility of migration of adsorbed atoms. The nature of $\sigma$ bonding is mainly determined by the hybridization of the $\mathrm{p}_{z}$ orbital of the $\mathrm{X}$ atom and the $\mathrm{s}-\mathrm{p}_{z}$ orbital of the first nearest neighbor $\mathrm{B}$ atom. This bonding character is more significant for the $\mathrm{F}$ atom case which in general differs from other halogens. Doping also modifies the electronic structure of borophene. However, borophene remains metallic (differing from the pristine case) and bands cross the Fermi level in all directions, mainly altering the anisotropic nature of electrical conductivity. 
Interestingly, for all cases, $\mathrm{X}_{2}$ molecules dissociate spontaneously and are atomically adsorbed on the nearest $\mathrm{T}_{\mathrm{u}}$ sites also confirming the high reactivity of borophene. The adsorption of halogen atoms on borophene extracts an excess amount of electrons from the antibonding states of triangular borophene which probably leads to the stabilization of the sheet. The obtained results are not only substantial for fundamental understanding of the interaction of halogen atoms with borophene but also suggest stable halogenated derivatives of borophene as a new class of material.

\section{Conflicts of interest}

There are no conflicts to declare.

\section{Acknowledgements}

The computational resources are provided by TUBITAK ULAKBIM, High Performance and Grid Computing Center (TR-Grid e-Infrastructure), and the National Center for High Performance Computing of Turkey (UHeM) under Grant No. 5003622015. This work was supported by the Scientific and Technological Research Council of Turkey (TUBITAK) under Project No. 115F088. E. D. acknowledges the financial support from the Turkish Academy of Sciences within the Outstanding Young Scientists Award Program (TUBA-GEBIP).

\section{References}

1 K. S. Novoselov, A. K. Geim, S. V. Morozov, D. Jiang, Y. Zhang, S. V. Dubonos, I. V. Grigorieva and A. A. Firsov, Science, 2004, 306, 666-669.

2 P. Vogt, P. De Padova, C. Quaresima, J. Avila, E. Frantzeskakis, M. C. Asensio, A. Resta, B. Ealet and G. Le Lay, Phys. Rev. Lett., 2012, 108, 155501.

3 M. Dávila, L. Xian, S. Cahangirov, A. Rubio and G. Le Lay, New J. Phys., 2014, 16, 095002.

4 F.-f. Zhu, W.-j. Chen, Y. Xu, C.-l. Gao, D.-d. Guan, C.-h. Liu, D. Qian, S.-C. Zhang and J.-f. Jia, Nat. Mater., 2015, 14, 1020-1025.

5 L. Li, Y. Yu, G. J. Ye, Q. Ge, X. Ou, H. Wu, D. Feng, X. H. Chen and Y. Zhang, Nat. Nanotechnol., 2014, 9, 372-377.

6 J. Ji, X. Song, J. Liu, Z. Yan, C. Huo, S. Zhang, M. Su, L. Liao, W. Wang, Z. Ni, Y. Hao and H. Zeng, Nat. Commun., 2016, 7, 13352 .

7 I. K. Drozdov, A. Alexandradinata, S. Jeon, S. Nadj-Perge, H. Ji, R. Cava, B. A. Bernevig and A. Yazdani, Nat. Phys., 2014, 10, 664-669.

8 A. J. Mannix, X.-F. Zhou, B. Kiraly, J. D. Wood, D. Alducin, B. D. Myers, X. Liu, B. L. Fisher, U. Santiago, J. R. Guest, M. J. Yacaman, A. Ponce, A. R. Oganov, M. C. Hersam and N. P. Guisinger, Science, 2015, 350, 1513-1516.

9 B. Feng, J. Zhang, Q. Zhong, W. Li, S. Li, H. Li, P. Cheng, S. Meng, L. Chen and K. Wu, Nat. Chem., 2016, 8, 563-568. 10 Z. Zhang, E. S. Penev and B. I. Yakobson, Nat. Chem., 2016, 8, 525-527.
11 T. Ogitsu, E. Schwegler and G. Galli, Chem. Rev., 2013, 113, 3425-3449.

12 X. Wu, J. Dai, Y. Zhao, Z. Zhuo, J. Yang and X. C. Zeng, ACS Nano, 2012, 6, 7443-7453.

13 D. Ciuparu, R. F. Klie, Y. Zhu and L. Pfefferle, J. Phys. Chem. B, 2004, 108, 3967-3969.

14 E. S. Penev, S. Bhowmick, A. Sadrzadeh and B. I. Yakobson, Nano Lett., 2012, 12, 2441-2445.

15 H.-J. Zhai, Y.-F. Zhao, W.-L. Li, Q. Chen, H. Bai, H.-S. Hu, Z. A. Piazza, W.-J. Tian, H.-G. Lu, Y.-B. Wu, Y.-W. Mu, G.-F. Wei, Z.-P. Liu, J. Li, S.-D. Li and L.-S. Wang, Nat. Chem., 2014, 6, 727-731.

16 H. Tang and S. Ismail-Beigi, Phys. Rev. Lett., 2007, 99, 115501.

17 E. S. Penev, S. Bhowmick, A. Sadrzadeh and B. I. Yakobson, Nano Lett., 2012, 12, 2441-2445.

18 C. Lee, X. Wei, J. W. Kysar and J. Hone, Science, 2008, 321, 385-388.

19 H. Sun, Q. Li and X. Wan, Phys. Chem. Chem. Phys., 2016, 18, 14927-14932.

20 B. Peng, H. Zhang, H. Shao, Y. Xu, R. Zhang and H. Zhu, J. Mater. Chem. C, 2016, 4, 3592-3598.

21 E. S. Penev, A. Kutana and B. I. Yakobson, Nano Lett., 2016, 16, 2522-2526.

22 M. Gao, Q.-Z. Li, X.-W. Yan and J. Wang, Phys. Rev. B, 2017, 95, 024505.

23 R. Xiao, D. Shao, W. Lu, H. Lv, J. Li and Y. Sun, Appl. Phys. Lett., 2016, 109, 122604.

24 S. H. Mir, S. Chakraborty, P. C. Jha, J. Wärnå, H. Soni, P. K. Jha and R. Ahuja, Appl. Phys. Lett., 2016, 109, 053903.

25 H. Jiang, Z. Lu, M. Wu, F. Ciucci and T. Zhao, Nano Energy, 2016, 23, 97-104.

26 B. Mortazavi, A. Dianat, O. Rahaman, G. Cuniberti and T. Rabczuk, J. Power Sources, 2016, 329, 456-461.

27 J. Wang, Y. Du and L. Sun, Int. J. Hydrogen Energy, 2016, 41, 5276-5283.

28 A. Criado, M. Melchionna, S. Marchesan and M. Prato, Angew. Chem., Int. Ed., 2015, 54, 10734-10750.

29 Q. Tang, Z. Zhou and Z. Chen, Nanoscale, 2013, 5, 4541-4583.

30 S. Cahangirov, H. Sahin, G. Le Lay and A. Rubio, Introduction to the physics of silicene and other $2 D$ materials, Springer, 2016, vol. 930.

31 F. Karlicky, K. Kumara Ramanatha Datta, M. Otyepka and R. Zboril, ACS Nano, 2013, 7, 6434-6464.

32 R. R. Nair, W. Ren, R. Jalil, I. Riaz, V. G. Kravets, L. Britnell, P. Blake, F. Schedin, A. S. Mayorov, S. Yuan, M. I. Katsnelson, H.-M. Cheng, W. Strupinski, L. G. Bulusheva, A. V. Okotrub, I. V. Grigorieva, A. N. Grigorenko, K. S. Novoselov and A. K. Geim, Small, 2010, 6, 2877-2884.

33 F. Karlicky, K. Kumara Ramanatha Datta, M. Otyepka and R. Zboril, ACS Nano, 2013, 7, 6434-6464.

34 H. Sahin, M. Topsakal and S. Ciraci, Phys. Rev. B: Condens. Matter Mater. Phys., 2011, 83, 115432.

35 Z. Wang, J. Wang, Z. Li, P. Gong, X. Liu, L. Zhang, J. Ren, H. Wang and S. Yang, Carbon, 2012, 50, 5403-5410.

36 B. Li, L. Zhou, D. Wu, H. Peng, K. Yan, Y. Zhou and Z. Liu, ACS Nano, 2011, 5, 5957-5961. 
37 J. Zheng, H.-T. Liu, B. Wu, C.-A. Di, Y.-L. Guo, T. Wu, G. Yu, Y.-Q. Liu and D.-B. Zhu, Sci. Rep., 2012, 2, 662.

38 H. Sahin and S. Ciraci, J. Phys. Chem. C, 2012, 116, 24075-24083.

39 O. Leenaerts, H. Peelaers, A. Hernández-Nieves, B. Partoens and F. Peeters, Phys. Rev. B: Condens. Matter Mater. Phys, 2010, 82, 195436.

40 D. Tristant, P. Puech and I. C. Gerber, J. Phys. Chem. C, 2015, 119, 12071-12078.

41 W. Li, S. Sheng, J. Chen, P. Cheng, L. Chen and K. Wu, Phys. Rev. B, 2016, 93, 155410.

42 Y. Xue, Q. Liu, G. He, K. Xu, L. Jiang, X. Hu and J. Hu, Nanoscale Res. Lett., 2013, 8, 49.

43 N. Gao, W. T. Zheng and Q. Jiang, Phys. Chem. Chem. Phys., 2012, 14, 257-261.

44 Y. Ma, Y. Dai, C. Niu and B. Huang, J. Mater. Chem., 2012, 22, 12587-12591.

45 Y. Ma, Y. Dai, M. Guo, C. Niu and B. Huang, J. Phys. Chem. C, 2012, 116, 12977-12981.

46 W. Kohn and L. J. Sham, Phys. Rev., 1965, 140, A1133-A1138.

47 P. Hohenberg and W. Kohn, Phys. Rev., 1964, 136, B864-B871.

48 P. E. Blöchl, Phys. Rev. B: Condens. Matter Mater. Phys., 1994, 50, 17953-17979.

49 G. Kresse and D. Joubert, Phys. Rev. B: Condens. Matter Mater. Phys., 1999, 59, 1758.

50 G. Kresse and J. Furthmüller, Comput. Mater. Sci., 1996, 6, 15-50.

51 G. Kresse and J. Furthmüller, Phys. Rev. B: Condens. Matter Mater. Phys., 1996, 54, 11169.
52 J. P. Perdew, K. Burke and M. Ernzerhof, Phys. Rev. Lett., 1996, 77, 3865.

53 S. Grimme, J. Comput. Chem., 2006, 27, 1787-1799.

54 G. Henkelman, A. Arnaldsson and H. Jónsson, Comput. Mater. Sci., 2006, 36, 354-360.

55 H. J. Monkhorst and J. D. Pack, Phys. Rev. B: Solid State, 1976, 13, 5188.

56 Total energies are corresponding to the Helmholtz free energy at zero temperature, for a constant volume and zero-point vibrations are neglected.

57 K. Reuter and M. Scheffler, Phys. Rev. B: Condens. Matter Mater. Phys., 2001, 65, 035406.

58 G. Mills, H. Jónsson and G. K. Schenter, Surf. Sci., 1995, 324, 305-337.

59 G. Henkelman and H. Jonsson, J. Chem. Phys., 2000, 113, 9978-9985.

60 A. Lherbier, A. R. Botello-Méndez and J.-C. Charlier, $2 D$ Mater., 2016, 3, 045006.

61 V. V. Kulish, Phys. Chem. Chem. Phys., 2017, 19, 11273-11281.

62 H. Tang and S. Ismail-Beigi, Phys. Rev. Lett., 2007, 99, 115501.

63 L.-C. Xu, A. Du and L. Kou, Phys. Chem. Chem. Phys., 2016, 18, 27284-27289.

64 X. Wang, H. Liu and S.-T. Tu, RSC Adv., 2015, 5, 6238-6245. 65 C. Xu, P. A. Brown, J. Lu and K. L. Shuford, J. Phys. Chem. C, 2015, 119, 17271-17277.

66 A. Rudenko, F. Keil, M. Katsnelson and A. Lichtenstein, Phys. Rev. B: Condens. Matter Mater. Phys., 2010, 82, 035427.

67 D. Alfè and M. Gillan, J. Chem. Phys., 2007, 127, 114709. 